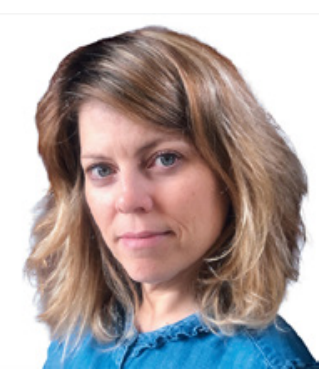

\title{
Challenges in Clinical Nutrition during the COVID-19 Pandemic
}

\author{
Christina N. Katsagoni \\ Agia Sofia Children's Hospital, Athens, Greece
}

Abstract from Thibault R, Coëffier M, Joly F, et al.: How the Covid-19 epidemic is challenging our practice in clinical nutrition - feedback from the field. Eur J Clin Nutr 2020;DOI:10.1038/s41430-020-00757-6

\begin{abstract}
The viral epidemic caused by the new Coronavirus SARS-CoV-2 is responsible for the new Coronavirus disease-2019 (Covid-19). Fifteen percent of the Covid-19 patients will require hospital stay, and $10 \%$ of them will need urgent respiratory and hemodynamic support in the intensive care unit (ICU). Covid-19 is an infectious disease characterized by inflammatory syndrome, itself leading to reduced food intake and increased muscle catabolism. Therefore Covid-19 patients are at high risk of being malnourished, making the prevention of malnutrition and the nutritional management key aspects of care. Urgent, brutal and massive arrivals of patients needing urgent respiratory care and artificial ventilation lead to the necessity to reorganize hospital care, wards and staff. In that context, nutritional screening and care may not be considered a
\end{abstract}

priority. Moreover, at the start of the epidemic, due to mask and other protecting material shortage, the risk of healthcare givers contamination have led to not using enteral nutrition, although indicated, because nasogastric tube insertion is an aerosol-generating procedure. Clinical nutrition practice based on the international guidelines should therefore adapt and the use of degraded procedures could unfortunately be the only way. Based on the experience from the first weeks of the epidemic in France, we emphasize ten challenges for clinical nutrition practice. The objective is to bring objective answers to the most frequently met issues to help the clinical nutrition caregivers to promote nutritional care in the hospitalized Covid-19 patient. We propose a flow chart for optimizing the nutrition management of the Covid-19 patients in the non-ICU wards.

(c) 2020 The Author(s) 


\section{Background}

Recent data show that COVID-19 patients are at high risk for malnutrition. The infection impairs immune function and causes a hypercatabolic state that induces circulating inflammatory cytokines leading to rapid muscle waste and decreased food intake.

Preventing malnutrition and giving the appropriate nutritional support is essential to those patients, as endorsed by the international guidelines of the European Society of Clinical Nutrition and Metabolism (ESPEN) [1] and the American Society for Parenteral and Enteral Nutrition (ASPEN) [2]. Still, it is important to know whether these guidelines are feasible in clinical practice during the COVID-19 pandemic.

\section{Review Results}

In the review of Thibault and colleagues in the European Journal of Clinical Nutrition [3], the researchers highlighted the risk of malnutrition and the key role of nutrition therapy in COVID-19 patients. What was interesting were the challenges they observed in their clinical nutrition practice when implementing international guidelines (e.g. ESPEN, ASPEN) during the first weeks of the pandemic in France. Concerning these challenges, they suggested a nutrition protocol in order to promote nutritional care in hospitalized nonICU COVID-19 patients (Fig. 1).

\section{Challenges for the Cinical Nutrition Practice during the} COVID-19 Pandemic

1 New clinical nutrition protocols are needed: As dietitians are often not allowed to visit COVID-19 (or even non-COVID-19) hospital wards, clinical nutrition teams need to write and implement new nutrition protocols in order to keep nutritional screening and evaluation a priority.

\section{The nutrition protocols need to be simple and practical:} Some healthcare professionals are often not aware of clinical nutrition guidelines. Thus, dietitians need to create simple and practical nutrition protocols based on feasible decision algorithms.

3 Simple indicators should be used to assess and diagnose malnutrition: If applicable, it is important to measure a patient's actual weight and height upon admission and calculate their recent weight loss and body mass index (BMI). This is to identify the ESPEN phenotypic and etiologic criteria for the diagnosis of malnutrition.

4 Energy and protein needs should be assessed in a simple manner: If available, indirect calorimetry should be used safely and appropriately. Alternatively, weight-based formulae or predictive equations should be used. Special consideration should be given in obese patients (BMI $\geq 30$ ) by using the adjusted body weight. 5 Healthcare professionals should be aware of refeeding syndrome: Every COVID-19 unit should have specific protocols for the prevention of refeeding syndrome.

6 Adjustments in hospital food delivery should be made in order to avoid insufficient food intake: Higher energy and protein foods should be delivered to patients upon admission along with two oral nutritional supplements every day. Nasogastric tubes (NGT) should be considered as a first line nutrition support in COVID-19 patients when oral food intake has fallen below $70 \%$ of target after $48 \mathrm{~h}$.

7 Misconceptions around the use of enteral nutrition (EN) should be fought: Based on the available evidence, the insertion of a NGT does not induce or worsen hypoxemia and apnoea. Once in place, it does not block natural ventilation or the oxygen mask position. Accordingly, the continuous feeding does not affect EN tolerance.

8 Factors that make EN unfeasible need to be known: In patients with high oxygen requirements, EN may be insufficient and thus parenteral nutrition (PN) should be considered. Strict antiviral protection rules must be followed in order to insert an NTG, and CT

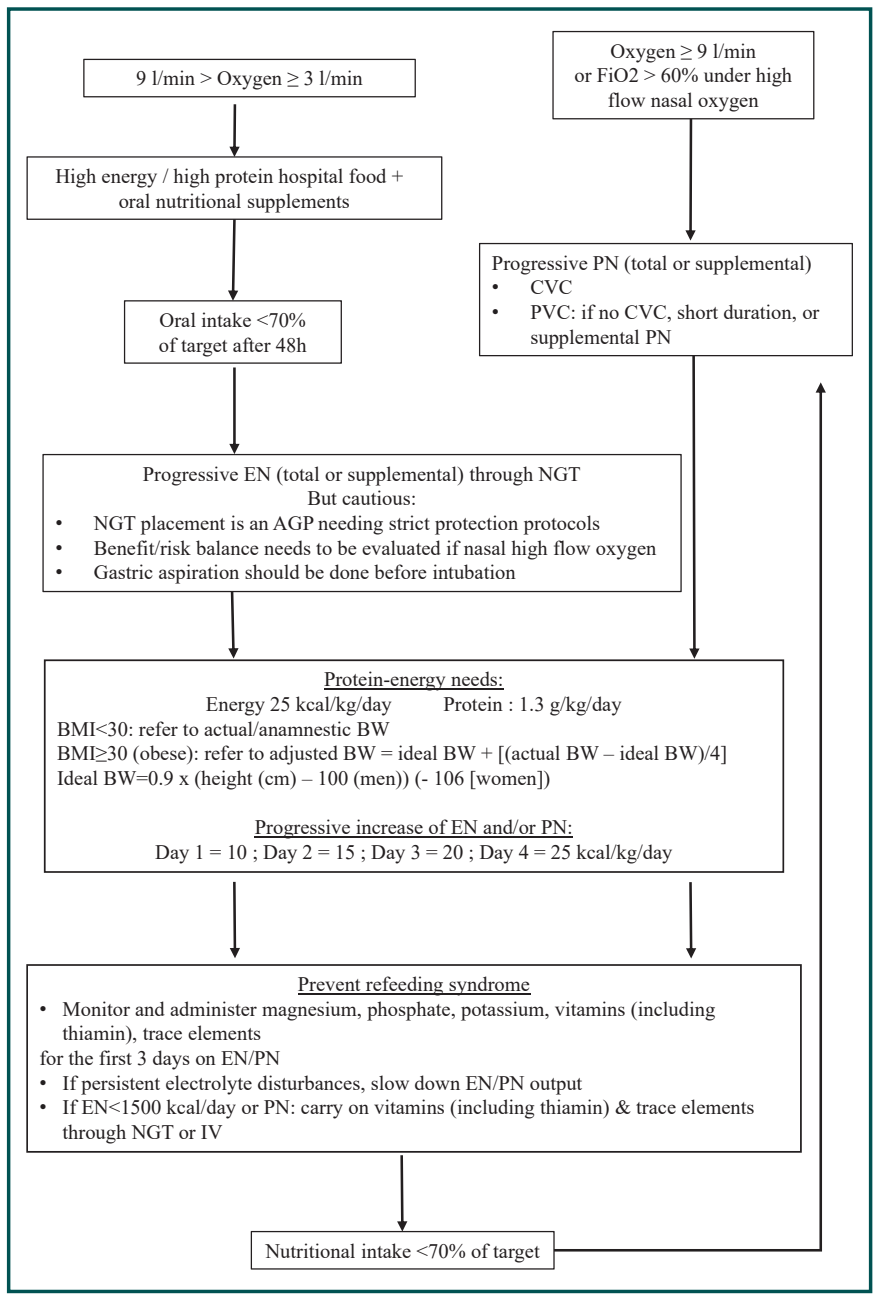

Fig. 1. Nutrition protocol for hospitalized COVID-19 patients in non-ICU wards (with permission from Thibault, et al. [3]). BMI = body mass index, $\mathrm{BW}=$ body weight, $\mathrm{CVC}=$ central venous catheter, $\mathrm{EN}=$ enteral nutrition, $I C U$ = intensive care unit, IV = intravenous, $N G T=$ nasogastric tube, $P N=$ parenteral nutrition, $\mathrm{PVC}=$ peripheral venous catheter. 
should be used to check the tube's location, in case a chest X-ray is not feasible. EN should be administrated via a pump with flow regulator. In case of a shortage, portable pumps should be available. Before an emergency intubation, a stomach aspiration via NGT might be necessary.

\section{PN may be considered in case EN is not feasible.}

10 Post-ICU patients should receive appropriate nutritional support at the rehabilitation phase: $A$ thorough nutritional evaluation and support is needed for post-ICU COVID-19 patients during the recovery phase.

\section{Personal Experience}

In Greece, the implementation of the international nutrition guidelines has been challenged in the daily clinical practice during the COVID-19 pandemic. Often, dietitians are not allowed to enter the COVID-19 wards. However, after the establishment of the nutrition support teams (that include doctors, nurses and pharmacists), which have been established in Greece since 2012, dietitians have the opportunity to collaborate with the other team members, in order to design individualized nutritional care plans for each patient. In specific cases, they have the opportunity to enter COVID-19 wards and use more sophisticated methods and measurements for the evaluation and management of the patients, e.g. indirect calorimetry, anthropometric measurements (circumferences, skin folds, etc).

\section{Disclosure Statement}

I hereby declare that there are no conflicts of interest with regard to this commentary.

\section{References}

1 Barazzoni R, Bischoff SC, Breda J, et al.: ESPEN expert statements and practical guidance for nutritional management of individuals with SARS-CoV-2 infection. Clin Nutr. 2020;39(6):1631-1638.

2 Martindale R, Patel J, Taylor B, et al.: Nutrition therapy in the patient with COVID-19 disease requiring ICU care. Nutrition Society of Critical Care Medicine and the American Society for Parenteral and Enteral Nutrition. 2020.

3 Thibault R, Coëffier M, Joly F, Bohé J, et al.: How the Covid-19 epidemic is challenging our practice in clinical nutrition-feedback from the field. Eur J Clin Nutr. 2020;DOI:10.1038/s41430-020-00757-6.

Correspondence: Christina N. Katsagoni, PhD, Agia Sofia Children's Hospital, Thivon \& Papadiamantopoulou, 11527 Athens, Greece, christina.katsagoni@gmail.com 